\title{
Assessing Accuracy and Precision of 3D Augmented Reality Holographic models derived from DICOM data
}

Jesus Uribe

University of California San Francisco

Lan $\mathrm{Vu}$

University of California San Francisco

Benjamin Laguna

University of California San Francisco

Jesse Courtier ( $\square$ jesse.courtier@ucsf.edu )

UCSF Benioff Children's Hospital Oakland https://orcid.org/0000-0003-2717-8653

\section{Technical Note}

Keywords: Augmented reality, holograms, DICOM, PACS, 3D AR, CT DICOM

Posted Date: June 1st, 2020

DOI: https://doi.org/10.21203/rs.3.rs-16722/v2

License: (c) (i) This work is licensed under a Creative Commons Attribution 4.0 International License. Read Full License 


\section{Abstract}

Objective: Assess accuracy and precision of measurements on 3D Augmented reality (AR) models derived from CT DICOM data, and compare AR model measurements with PACS measurements.

Materials/Methods: 5 individual 3D hologram models were produced using a CT phantom with fiducial markers set at varying distances. DICOM files were translated into 3D AR models using open source software. AR models were adapted for display on an AR device using a novel application. AR models were projected and distances between the projected fiducial markers were measured. Finally, 5 measurements each were obtained of the holographic projected distances between fiducials in the $\mathrm{x} 1, \mathrm{y} 1$, and $\mathrm{z} 1$ labeled planes respectively for precision assessment. Mann-Whitney $\mathrm{U}$ test was performed to compare measured distances on AGFA-PACS, AR models, and actual measured distances on phantom models.

Results: No significant difference was found between gold standard measurements and either PACS measurements $(p=0.9124)$ or AR measurements $(p=0.8966)$. AR model measurements had a standard error of $0.24 \mathrm{~mm}, 0.24 \mathrm{~mm}$, and $0.38 \mathrm{~mm}$ in the $x, y$, and $z$ planes respectively. Furthermore, measurements on AR models demonstrated a high degree of accuracy in comparison to gold standard measurements.

Conclusion: Current AR technology is can produce reliable 3D AR models from CT DICOM data

\section{Introduction}

Augmented reality applications are being increasingly utilized in a number of fields including military, industry, and sports 1-3 with emerging potential applications in medical imaging 4-7. Recent advances in technology have allowed for increased portability of augmented reality hardware, making more widespread utilization possible 8,9. While preliminary investigation of accuracy and precision of augmented reality models has been described with large, projection based equipment 10 , only limited data exists for accuracy and precision of head mounted display (HMD)-based conversion of Digital Imaging and Communications in Medicine (DICOM) medical imaging information into augmented reality models (7). The aim of this study was to assess the accuracy and precision of the DICOM-derived 3D holographic models data on a HMD using a proprietary C\# programming language-based software application and to determine if any statistically significant differences exist between gold-standard physical measurements, Picture Archiving and Communication System (PACS)-based measurements, and Augmented Reality holographic measurements.

\section{Methods}

Five unique 3D models were produced using a CT quality control phantom (model 137856101, GE Healthcare, Waukesha WI USA) with fiducial markers (CT/MRI 2 mm center hole Multi-modality marker, MM3002, Izi Medical Products, Owings Mills, MD USA). The fiducial markers had an adhesive to allow for 
adjustment on the surface of the CT phantom. For each trial of the CT phantom, fiducial markers were adjusted to provide new distances in each of the orthogonal directions.

The distances were measured using electronic digital calipers (model 01407A, Neiko Tools, Henan, China). These measurements were set as the gold standard (GS), aiming to reproduce measurements gathered in clinical settings such as those via surgical ruler or calipers. The calipers' product information reports precise measurements with resolution of $0.01 \mathrm{~mm}$ and accurate measurements to $0.02 \mathrm{~mm}$. FIGURES 1,2. A total of six measurements between fiducial markers were made for each model CT phantom: two in the $x$-direction, two in the y-direction and two in the z-direction by one observer. The observer was trained to measure the shortest distance between fiducial markers set in the orthogonal directions $(x, y, z)$ using the inside calipers.

CT scans of the phantom were obtained using the Head CT quality control phantom settings $(5 \mathrm{~mm}$ slice thickness, $134 \mathrm{mAs}, 22.7 \mathrm{~cm}$ DFOV, $0.516: 1$ pitch) with $0.625 \mathrm{~cm} \times 0.625 \mathrm{~cm} \times 0.625 \mathrm{~cm}$ voxels on a GE Lightspeed CT scanner (GE Healthcare, Waukesha WI USA) and stored as DICOM files within our AGFA IMPAX version 6.7.0.3502 "site" Picture Archiving and Communication System (PACS), (AGFA, Mortsel, Belgium). The DICOM files obtained were converted into 3D phantom models, and measurements were made on the AGFA-PACS. The observer was trained to measure the shortest distance between the outer edges fiducial markers using only the "ruler" found within PACS. FIGURE 3.Additionally, the observer had access to axial, sagittal and coronal views to project views of fiducial markers in their most appropriate planes.

Open-source software programs Horos (Purview, Annapolis, MD USA) and Blender (Stichting Blender Foundation, Amsterdam, Netherlands) were used to translate DICOM files into a 3D image. The 3D images were adapted and loaded onto the AR HMD platform using a C\# programming language-based code on the Unity Platform (Unity Technologies, San Francisco, CA USA). The AR HMD used for this study was the Hololens 1 (Microsoft, Redmond, WA USA) OS version 10.0.14393.1358. The settings for HoloLens projections were set to default factory settings with the exception of distance of initial hologram projection set to $30 \mathrm{~cm}$ from HoloLens. This was done to project the hologram within approximately arm's length of the user. The hologram was manipulated via rotation and translation without scaling size to obtain best views of markers. FIGURE 4 (with supplemental video) demonstrates a side-by-side comparison of the CT phantom and its hologram. The 3D models were projected as holograms and the distances between the projected fiducial markers were measured by the same observer as GS measurements. Using the built-in AR HMD capabilities, the hologram was pinned to a table where the calipers could be laid flatly to make the measurements, FIGURE 5 . These measurements are known as Holo measurements. 
A power analysis was performed using SAS University Edition (SAS Institute Inc., Cary, NC). This demonstrated that for paired t-test $(\mathbb{\nabla}=0.05)$ with a sample size of $n=30$ per group, the power would be $80 \%$ in detecting a difference of $0.3 \mathrm{~mm}$. In a previous study assessing accuracy and reliability of CT measurement, max difference was observed in this range (11); the authors opted to detect a difference of this magnitude using this sample size in consideration of available time, resources and training in both PACS and holo measurements.

Since DICOM and PACS have become standard in the clinical radiology workspace, the assessment of accuracy and precision of the Holo measurements were set against this standard (12). To determine accuracy, the absolute error from the GS was gathered for PACS and Holo measurements. To compare the means and distributions of the absolute error, a two-tailed t-test was employed. For the assessment of precision, a right-tailed chi-square test of variance was used, where the PACS absolute error variance is set as the null. Additionally, a root-mean-square error was calculated for a series of repeated measurements made in each orthogonal direction $(n=15)$.

\section{Results}

All measurements taken for each CT phantom trial on GS, PACS, and Hologram are found in Table 1.

The GS distances measurements are summarized as follows: $\min =10.4 \mathrm{~mm}, \mathrm{Q} 1=21.9 \mathrm{~mm}, \mathrm{Q} 2=37.7 \mathrm{~mm}$, $\mathrm{Q} 3=51.7 \mathrm{~mm}, \max =87.8 \mathrm{~mm}$ and $\mathrm{mean}=38.7 \mathrm{~mm}$. For PACS, the measurement values obtained were: $\min =11.3 \mathrm{~mm}, \mathrm{Q} 1=21.7 \mathrm{~mm}, \mathrm{Q} 2=37.5 \mathrm{~mm}, \mathrm{Q} 3=52.1 \mathrm{~mm}, \mathrm{max}=88.4 \mathrm{~mm}$ and mean $=39.0 \mathrm{~mm}$. Holo measurement values were: $\min =10.8 \mathrm{~mm}, \mathrm{Q} 1=22.0 \mathrm{~mm}, \mathrm{Q} 2=37.5 \mathrm{~mm}, \mathrm{Q} 3=50.9 \mathrm{~mm}$, $\mathrm{max}=87 \mathrm{~mm}$ and mean $=34.5 \mathrm{~mm}$.

A two sample t-test comparing PACS and Holo absolute error revealed no statistically significant difference [ $\mathrm{M}_{\text {PACS }} 0.55 \mathrm{~mm} \pm .0 .39$ vs $\left.\mathrm{M}_{\text {Holo }} 0.59 \mathrm{~mm} \pm 0.45 ; p=0.75\right]$ Table 2. A right-tailed chi-square test of variance demonstrated no significant difference in absolute error of Holo measurements compared to the PACS: $X^{2}(29, N=30)=38.81, p=.1054$. The RMS was $0.783 \mathrm{~mm}$ across repeated Holo measurements for one phantom trial. For the repeated measures, the Shapiro-Wilk test was performed and demonstrated there no significant difference from a normal distribution $(p=0.713)$

\section{Discussion}


Currently, there is little existing literature assessing the accuracy of the 3D hologram derived from DICOM data.There have been some studies investigating the reliability of applications of AR in the medical workspace. A 2017 study evaluated the utility of AR in endovascular interventions using the HMD (7). CT data was utilized to reproduce a hologram of vasculature superimposed on a phantom, and a two-step calibration employing a tracked catheter demonstrated the plausibility of visualizing endovascular procedures without the use of $x$-ray. Their accuracy assessment pertained to the first calibration step and demonstrated a root-mean-square error of $4.357 \mathrm{~mm}$ in 20 trials of 4 landmarks. Though there is no distinction on the error and its dimensionality, our data suggest a smaller degree of error possibly due to the scale of our phantom.

The results of this study suggest that Holo measurements are as accurate as those made on the PACS. The Holo measurements trended towards less precise than those measurements made on PACS, however not at a significant level. This may be explained in part due to the lack tactile-feedback of holograms, leading to less precise measurements.

Several limitations were faced in obtaining measurements across the modalities. The GS instrument's intrinsic errors were ignored as additional sources of error. Given that most clinical measurements are rarely recorded to the hundredth of a millimeter, the authors deemed these errors less relevant for the purpose of this study. Additionally, user setting variability on the HMD was not assessed and would be a limitation of this study. A challenge faced while obtaining measurements on the Holo models was that distances were made by visually estimating distances between markers. On the other hand, GS measurements on the physical model had tactile-feedback to establish edges, while the Holo models were reliant on the observer's best estimation of depth and distance between markers. Furthermore, there was no assessment for variability between HMD users and no consideration for user experience which may impact accuracy and precision of measurements.

Ultimately, this study demonstrates the utility and reliability of AR HMD technology and for rendering accurate holographic models. The advantage of holographic models is the visualization of a 3D internal anatomical structures and the ability to superimpose the image on a real environment. The AR holographic models have potential to directly impact fields of medicine such as radiology, surgery and medical education.

\section{Conclusion}

Measurements on hologram models demonstrated a high degree of accuracy compared with reference standard measurements, nearly approaching that of PACS measurements. The precision of Hologram 
measurements warrant further investigation, especially their reproducibility among different users. Current augmented reality technology can produce reliable 3D holograms from CT DICOM data and could be used for educational, training, or research purposes. Our study provides the groundwork for future larger-scale research into AR's emerging applications in medicine.

\section{Declarations}

\section{ACKNOWLEDGEMENTS:}

1. Preliminary findings of this work were presented at the 2018 Annual Meeting of the American Roentgen Ray Society, Washington, D.C.

2. Microsoft HoloLens v1 used with permission from Microsoft (this equipment was purchased and owned by the senior author).

Declarations

- Ethics approval and consent to participate

Study was approved by our institutional review board.

- Consent for publication

Not applicable

- Availability of data and material

The datasets during and/or analysed during the current study available from the corresponding author on reasonable request.

- Competing interests

JU: Jesus Uribe -Nothing to disclose

LV: Lan Vu, MD -Nothing to disclose

BL: Benjamin Laguna, MD -Founder, Sira Medical, pre-revenue Augmented Reality startup, not discussed in this manuscript

JC: Jesse Courtier, MD - Founder, Sira Medical, pre-revenue Augmented Reality startup, not discussed in this manuscript

- Funding 
CTSIWork was funded by the CTSI (Clinical and Translational Science Institute) \& UCSF Catalyst Program of Innovation Ventures Fund

- Authors' contributions

Author Contributions

1. Guarantor of the integrity of the entire study: JC

2. Study concepts and design: JC

3. Literature research: JU, BL

4. Clinical Studies: $n / a$

5. Experimental studies/data analysis: JU, JC

6. Statistical analysis: LV

7. Manuscript preparation: JU, LV, BL

8. Manuscript editing: JU, LV, BL, JC

JU: Jesus Uribe

LV: Lan Vu, MD

BL: Benjamin Laguna, MD

JC: Jesse Courtier, MD

- Acknowledgements

none

\section{References}

1. Cui N, Kharel P, Gruev V. Augmented reality with Microsoft HoloLens holograms for near infrared fluorescence based image guided surgery. Molecular-Guided Surgery: Molecules, Devices, and Applications III. 2017; https://doi.org/10.1117/12.2251625.

2. Fernández-Caramés T, Fraga-Lamas P, Suárez-Albela M, Vilar-Montesinos M. A Fog Computing and Cloudlet Based Augmented Reality System for the Industry 4.0 Shipyard. Sensors 2018;18(6):1798.

3. Park J, Park J. 3DOF tracking accuracy improvement for outdoor Augmented Reality. 2010 IEEE International Symposium on Mixed and Augmented Reality. 2010; https://doi.org/10.1109/ismar.2010.5643598.

4. Fotouhi $\mathrm{J}$, Alexander $\mathrm{CP}$, Unberath $\mathrm{M}$, et al. Plan in 2-D, execute in 3-D: an augmented reality solution for cup placement in total hip arthroplasty. J Med Imaging (Bellingham) 2018;5(2):021205. 
5. Li X, Yi W, Chi H-L, Wang X, Chan APC. A critical review of virtual and augmented reality (VR/AR) applications in construction safety. Autom Constr 2018;86:150-62.

6. Teber D, Guven S, Simpfendörfer T, et al. Augmented reality: a new tool to improve surgical accuracy during laparoscopic partial nephrectomy? Preliminary in vitro and in vivo results. Eur Urol 2009;56(2):332-8.

7. Kuhlemann I, Kleemann M, Jauer P, Schweikard A, Ernst F. Towards X-ray free endovascular interventions - using HoloLens for on-line holographic visualisation. Healthcare Technology Letters 2017;4(5):184-7.

8. Tepper OM, Rudy HL, Lefkowitz A, et al. Mixed Reality with HoloLens: Where Virtual Reality Meets Augmented Reality in the Operating Room. Plast Reconstr Surg 2017;140(5):1066-70.

9. Kim Y, Kim H, Kim YO. Virtual Reality and Augmented Reality in Plastic Surgery: A Review. Arch Plast Surg 2017;44(3):179-87.

10. Low D, Lee CK, Dip LLT, Ng WH, Ang BT, Ng I. Augmented reality neurosurgical planning and navigation for surgical excision of parasagittal, falcine and convexity meningiomas. $\mathrm{Br} \mathrm{J}$ Neurosurg 2010;24(1):69-74.

11. Kim G, Jung HJ, Lee HJ, et al. Accuracy and Reliability of Length Measurements on ThreeDimensional Computed Tomography Using Open-Source OsiriX Software. J Digit Imaging 2012; 25:486-491.

12. Kahn CE Jr, Langlotz CP, Channin DS, Rubin DL. Informatics in radiology: an information model of the DICOM standard. Radiographics. 2011;31(1):295-304

\section{Tables}

Please see the supplementary files section to view the tables.

\section{Figures}




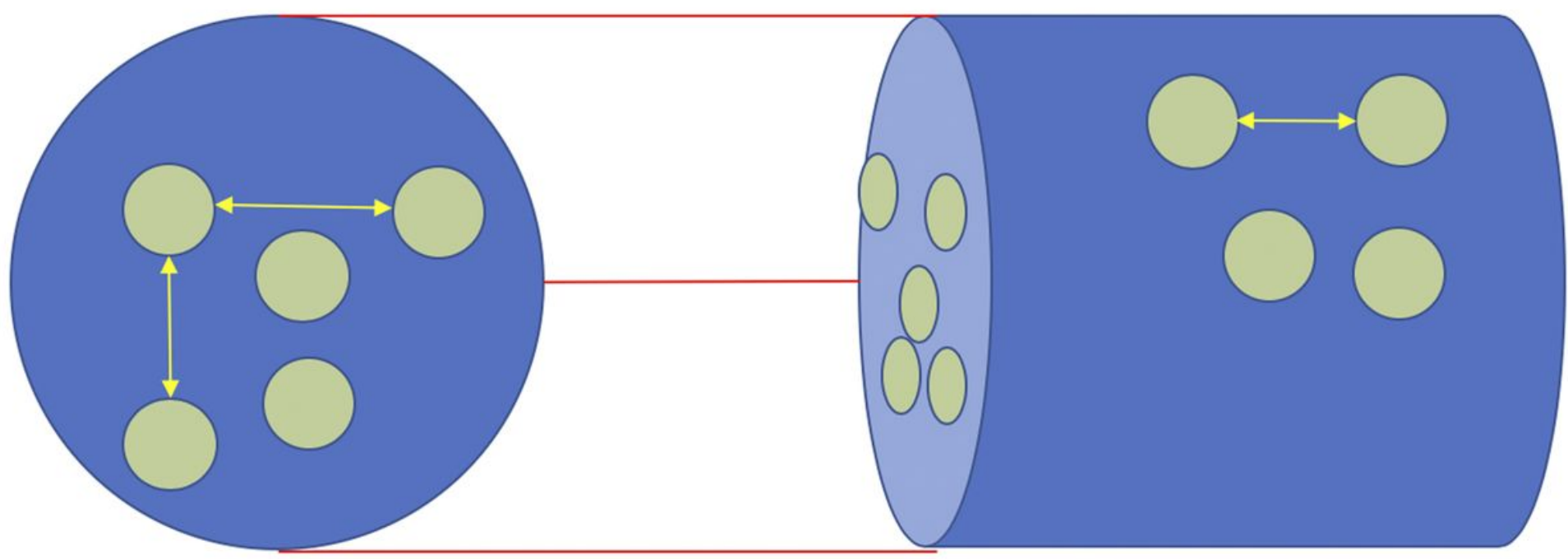

Figure 1

A representation of a trial demonstrating fiducial marker placement (light green circles) on the front (A) and side (B) of the CT quality control phantom.

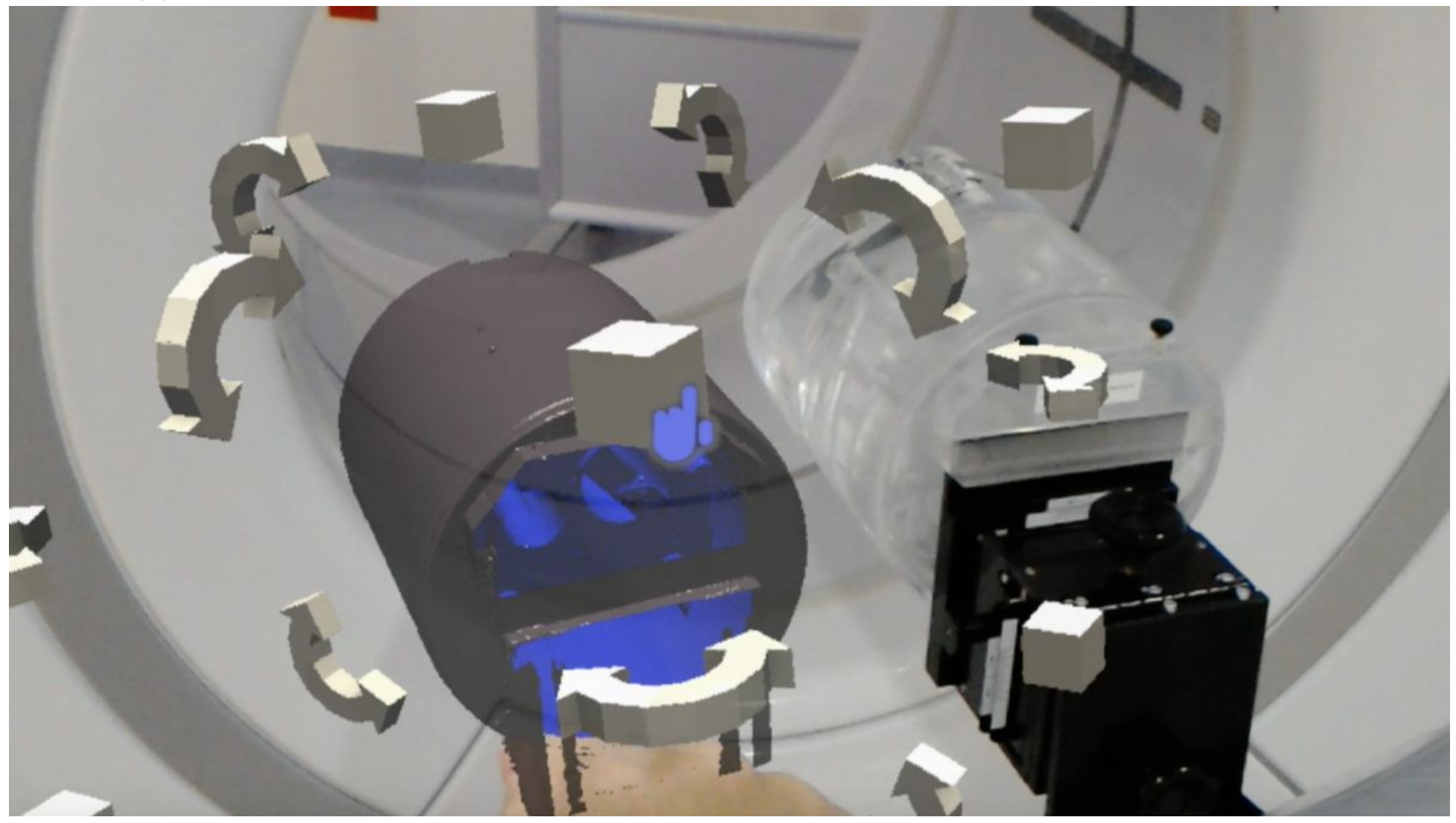

\section{Figure 2}

Photograph illustrating scientific caliper measuring distance between fiducial markers. Note: The top smaller calipers (depicted with yellow arrows) are used for this study. 


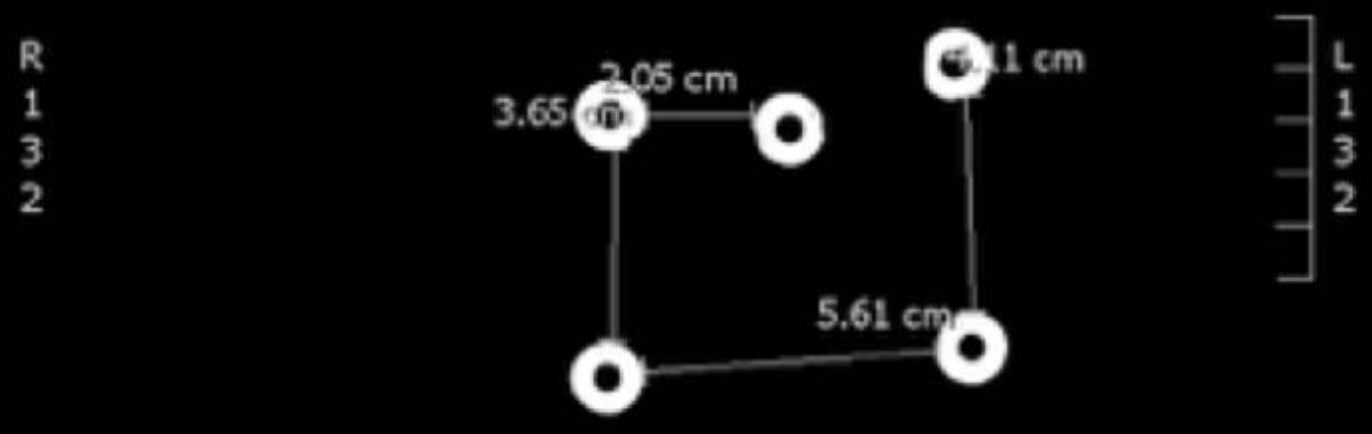

\section{Figure 3}

PACS ruler tool measuring distances between markers on DICOM image of scanned quality control phantom.

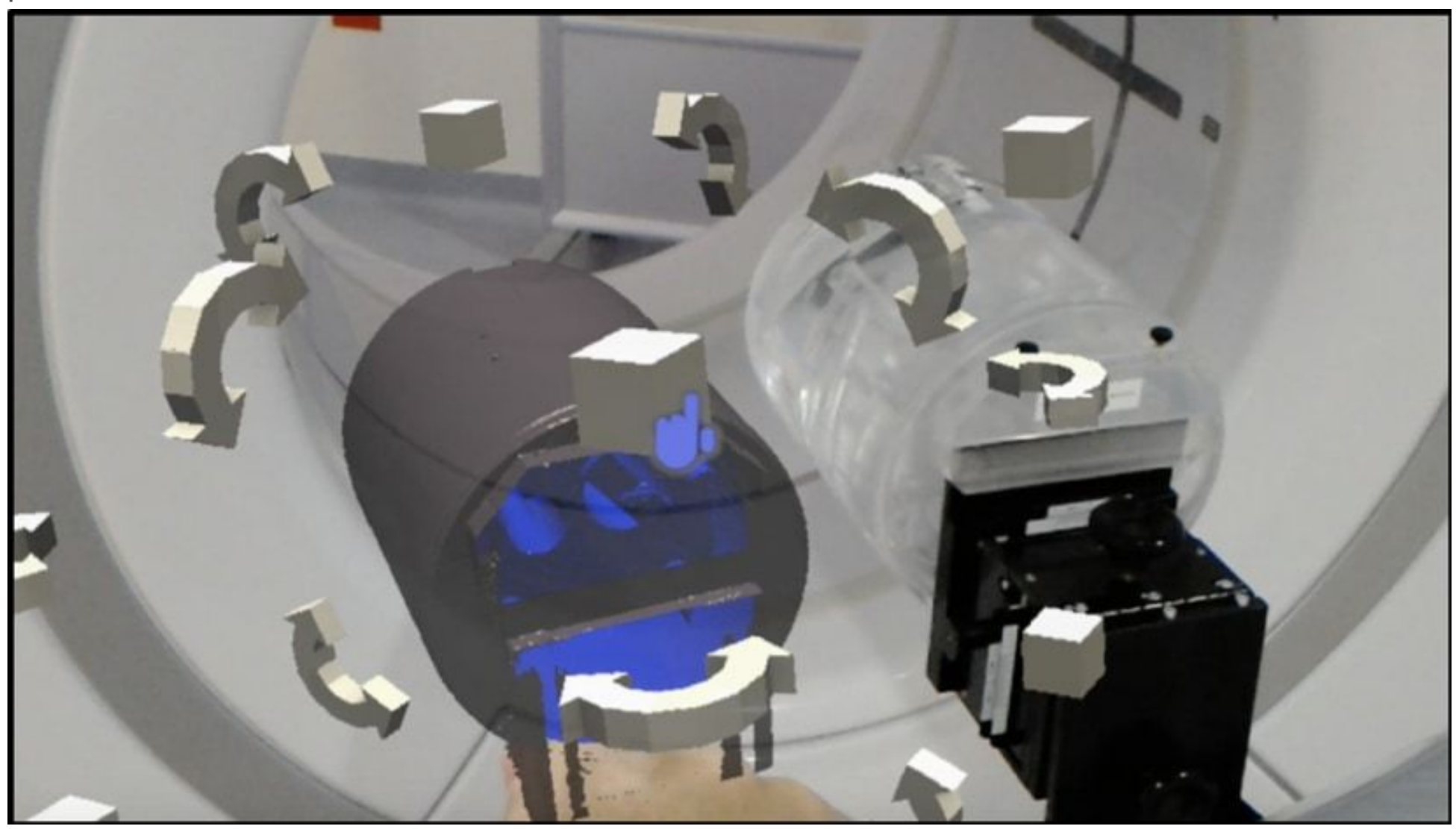

Figure 4 
Side-by-side comparison of CT phantom and the resulting Augmented Reality hologram (supplemental video file).

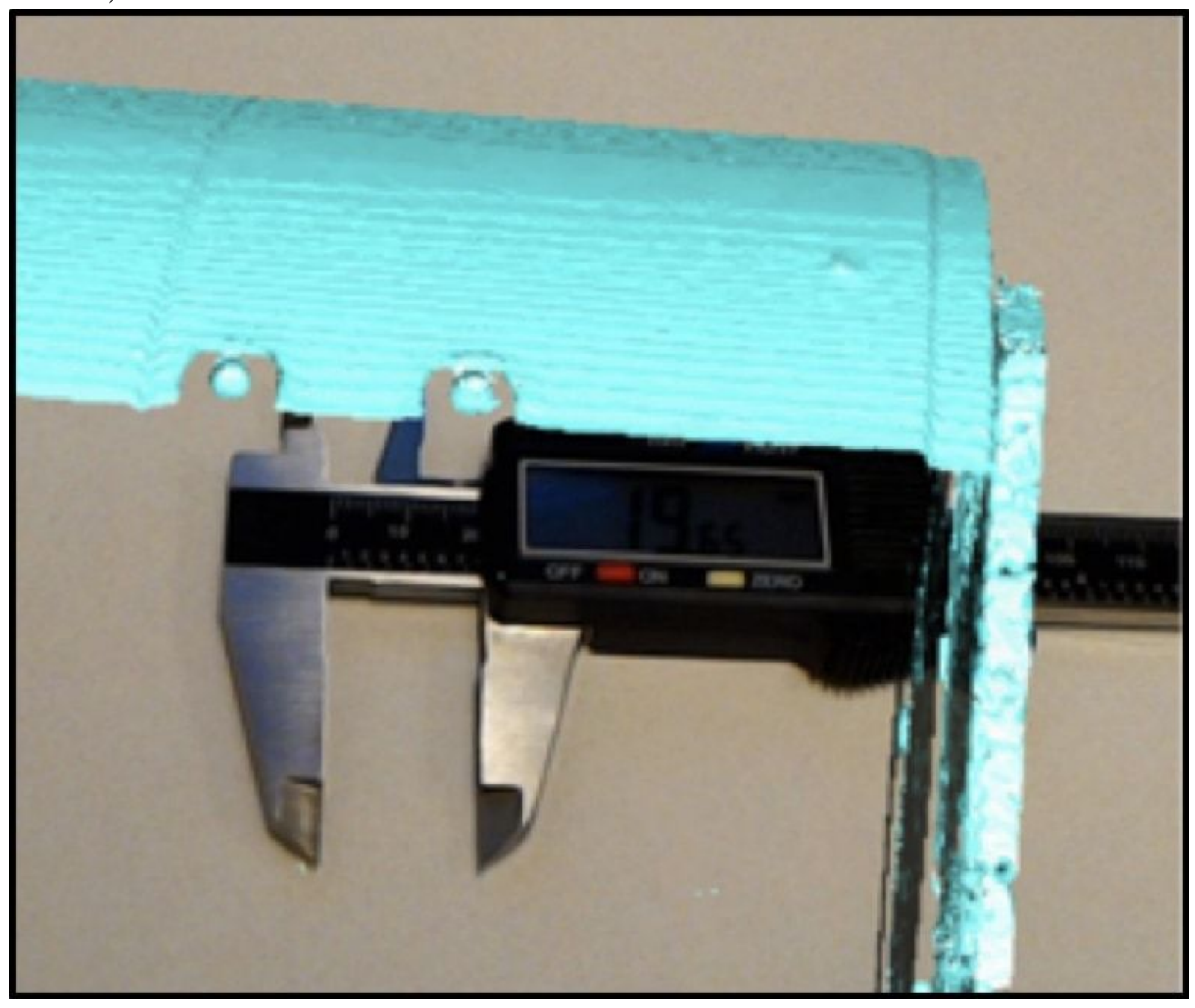

\section{Figure 5}

HMD screen capture taken of the hologram, top smaller calipers measuring the distance be markersraised circles on phantom model.

\section{Supplementary Files}

This is a list of supplementary files associated with this preprint. Click to download.

- Tables.docx 